ORIGINAL ARTICLE

\section{Abrocitinib versus Placebo or Dupilumab for Atopic Dermatitis}

T. Bieber, E.L. Simpson, J.I. Silverberg, D. Thaçi, C. Paul, A.E. Pink, Y. Kataoka, C.-Y. Chu, M. DiBonaventura, R. Rojo, J. Antinew, I. Ionita, R. Sinclair, S. Forman, J. Zdybski, P. Biswas, B. Malhotra, F. Zhang, and H. Valdez, for the JADE COMPARE Investigators*

A BSTRACT

\section{BACKGROUND}

The oral Janus kinase 1 (JAK1) inhibitor abrocitinib, which reduces interleukin-4 and interleukin-13 signaling, is being investigated for the treatment of atopic dermatitis. Data from trials comparing JAK1 inhibitors with monoclonal antibodies, such as dupilumab, that block interleukin-4 receptors are limited.

\section{METHODS}

In a phase 3, double-blind trial, we randomly assigned patients with atopic dermatitis that was unresponsive to topical agents or that warranted systemic therapy (in a 2:2:2:1 ratio) to receive $200 \mathrm{mg}$ or $100 \mathrm{mg}$ of abrocitinib orally once daily, $300 \mathrm{mg}$ of dupilumab subcutaneously every other week (after a loading dose of $600 \mathrm{mg}$ ), or placebo; all the patients received topical therapy. The primary end points were an Investigator's Global Assessment (IGA) response (defined as a score of 0 [clear] or 1 [almost clear] on the IGA [scores range from 0 to 4], with an improvement of $\geq 2$ points from baseline) and an Eczema Area and Severity Index-75 (EASI-75) response (defined as $\geq 75 \%$ improvement from baseline in the score on the EASI [scores range from 0 to 72]) at week 12. The key secondary end points were itch response (defined as an improvement of $\geq 4$ points in the score on the Peak Pruritus Numerical Rating Scale [scores range from 0 to 10]) at week 2 and IGA and EASI-75 responses at week 16.

\section{RESULTS}

A total of 838 patients underwent randomization; 226 patients were assigned to the 200-mg abrocitinib group, 238 to the $100-\mathrm{mg}$ abrocitinib group, 243 to the dupilumab group, and 131 to the placebo group. An IGA response at week 12 was observed in $48.4 \%$ of patients in the 200 -mg abrocitinib group, $36.6 \%$ in the 100 $\mathrm{mg}$ abrocitinib group, $36.5 \%$ in the dupilumab group, and $14.0 \%$ in the placebo group ( $\mathrm{P}<0.001$ for both abrocitinib doses vs. placebo); an EASI-75 response at week 12 was observed in $70.3 \%, 58.7 \%, 58.1 \%$, and $27.1 \%$, respectively $(\mathrm{P}<0.001$ for both abrocitinib doses vs. placebo). The 200-mg dose, but not the 100-mg dose, of abrocitinib was superior to dupilumab with respect to itch response at week 2 . Neither abrocitinib dose differed significantly from dupilumab with respect to most other key secondary end-point comparisons at week 16. Nausea occurred in $11.1 \%$ of the patients in the $200-\mathrm{mg}$ abrocitinib group and $4.2 \%$ of those in the 100 -mg abrocitinib group, and acne occurred in $6.6 \%$ and $2.9 \%$, respectively.

\section{CONCLUSIONS}

In this trial, abrocitinib at a dose of either $200 \mathrm{mg}$ or $100 \mathrm{mg}$ once daily resulted in significantly greater reductions in signs and symptoms of moderate-to-severe atopic dermatitis than placebo at weeks 12 and 16. The 200-mg dose, but not the 100 -mg dose, of abrocitinib was superior to dupilumab with respect to itch response at week 2. Neither abrocitinib dose differed significantly from dupilumab with respect to most other key secondary end-point comparisons at week 16 . (Funded by Pfizer; JADE COMPARE ClinicalTrials.gov number, NCT03720470.)
The authors' full names, academic degrees, and affiliations are listed in the Appendix. Address reprint requests to Dr. Valdez at Pfizer, 235 E. 42nd St., New York, NY 10017, or at hernan.valdez@ pfizer.com.

*A complete list of the investigators in the JADE COMPARE trial is provided in the Supplementary Appendix, available at NEJM.org.

N Engl J Med 2021;384:1101-12. DOI: 10.1056/NEJMoa2019380 Copyright () 2021 Massachusetts Medical Society. 
TOPIC DERMATITIS IS A COMMON, chronic inflammatory skin disease associated with itch, pain, sleep disturbance, anxiety, depression, and increased health care utilization. ${ }^{1,2}$ When topical therapies are insufficient, treatment with systemic agents, including dupilumab and immunosuppressants, has been recommended. ${ }^{3}$ Janus kinase (JAK) inhibitors, such as baricitinib, abrocitinib, and upadacitinib, are being investigated as systemic treatments for atopic dermatitis. ${ }^{4-8}$ Dupilumab, a subcutaneously administered anti-interleukin-4-receptor $\alpha$ monoclonal antibody, has been approved for the treatment of atopic dermatitis on the basis of randomized, controlled trials that showed that the medication (as monotherapy vs. placebo or in combination with topical glucocorticoids vs. placebo in combination with topical glucocorticoids) resulted in greater reductions in signs and symptoms of the disorder. ${ }^{9-14}$

Abrocitinib, a small-molecule JAK1 inhibitor that is administered orally once daily, inhibits signaling of interleukin-4, interleukin-13, and other cytokines involved in the pathogenesis of atopic dermatitis. ${ }^{15}$ Abrocitinib is less likely to stimulate an immunogenic response than biologic treatment. ${ }^{16,17}$ Although data from head-tohead trials with other JAK inhibitors are lacking, a phase $2 \mathrm{~b}$ trial and two phase 3 placebo-controlled trials have shown that 12 weeks of monotherapy with abrocitinib resulted in better outcomes in patients with atopic dermatitis than placebo. ${ }^{6-8}$ The primary objective of the JAK1 Atopic Dermatitis Efficacy and Safety (JADE) COMPARE trial was to evaluate the efficacy of abrocitinib, as compared with placebo, at 12 weeks in patients with moderate-to-severe atopic dermatitis who were receiving background topical therapy. A key secondary objective was to evaluate the efficacy of abrocitinib, as compared with placebo and with dupilumab (an active comparator in another drug class), on the basis of a reduction in itch at 2 weeks.

\section{METHODS}

TRIAL DESIGN AND OVERSIGHT

This was a multicenter, randomized, double-blind, double-dummy, placebo-controlled trial evaluating the efficacy and safety of two abrocitinib doses in adults with moderate-to-severe atopic dermatitis who were receiving background topi- cal therapy. Patients were enrolled in 18 countries (Australia and countries across North and South America, Europe, and Asia) from October 29, 2018, to August 5, 2019.

Patients entered a 28-day screening period, during which systemic and topical medications for atopic dermatitis were discontinued before the first dose of a trial medication or placebo was administered. Emollients were used twice daily, starting at least 7 days before randomization and continued throughout the trial, and therapy with a topical medication (applied once daily) was started on day 1 of the treatment period (baseline). Topical therapies that were allowed during the trial included low- or mediumpotency topical glucocorticoids, topical calcineurin inhibitors, and topical phosphodiesterase 4 inhibitors; patients were allowed to use more than one topical agent. Rescue therapy with systemic medications or topical treatments was not permitted if it included agents that were more potent than the allowed low- or medium-potency agents. The investigators, who were unaware of the trial-group assignments, evaluated efficacy and safety by means of a telephone call at week 1 and in-person visits at weeks $2,4,8,12,16,18$, and 20. Patients who completed the trial were allowed to enter a long-term phase 3 extension trial (ClinicalTrials.gov number, NCT03422822).

The trial was conducted in accordance with the principles of the Declaration of Helsinki and the International Council for Harmonisation Good Clinical Practice guidelines. All local regulatory requirements were followed. This research was approved by the institutional review board or ethics committee at each trial site. All patients provided written informed consent. An external data monitoring committee reviewed safety data throughout the trial.

The trial sponsor (Pfizer) designed the trial with input from the fourth author. The sponsor collected and analyzed the data and provided the drugs and placebo. The first draft of the manuscript was written by the last author, and medical writing and editorial support with subsequent drafts (funded by the sponsor and conducted in accordance with Good Publication Practice guidelines) was provided by ApotheCom, a medical communications company, under the guidance of the authors. The analysis of the data was performed by two authors employed by the sponsor and by the sponsor's programming group. All 
the authors vouch for the accuracy and completeness of the data, for the complete reporting of adverse events, and for the fidelity of the trial to the protocol (available with the full text of this article at NEJM.org). Confidentiality agreements were in place between the authors and the sponsor.

\section{PATIENTS}

Patients were eligible to participate if they were 18 years of age or older and had at least a 1-year history of atopic dermatitis that was moderate to severe at baseline, as determined by a score of 3 or higher on the Investigator's Global Assessment (IGA; scored on a 5-point scale [0, clear; 1 , almost clear; 2, mild; 3, moderate; and 4, severe] $)^{18}$; a score of at least 16 on the Eczema Area and Severity Index (EASI; scores range from 0 to 72, with higher scores indicating greater severity $)^{19}$; at least $10 \%$ body-surfacearea involvement; and a score of at least 4 on the Peak Pruritus Numerical Rating Scale (PP-NRS; scores range from 0 to 10 , with higher scores indicating greater pruritus). ${ }^{20}$ The PP-NRS was used with permission from Regeneron Pharmaceuticals and Sanofi, which developed the scoring system. During the 6 months before screening, all the patients had an inadequate response to topical medications that were given for at least 4 weeks or a need for systemic therapy to control their disease. Patients who had previously used systemic JAK inhibitors or dupilumab or had a medical history of conditions associated with thrombocytopenia, coagulopathy, or platelet dysfunction were ineligible. Patients with a history of conjunctivitis or similar eye conditions were not excluded. A complete list of inclusion and exclusion criteria is provided in the protocol.

\section{RANDOMIZATION}

Patients were randomly assigned in a 2:2:2:1 ratio to receive $200 \mathrm{mg}$ or $100 \mathrm{mg}$ of abrocitinib orally once daily, $300 \mathrm{mg}$ of dupilumab subcutaneously every other week (after a loading dose of $600 \mathrm{mg}$ ), or placebo for 16 weeks. The patients, investigators, and representatives of the sponsor were unaware of the trial-group assignments. Additional information on dosing and concomitant topical therapies is provided in Section S3 of the Supplementary Appendix, available at NEJM.org.

\section{END POINTS}

The primary end points were an IGA response (defined as a score of 0 or 1 on the IGA, with an improvement of $\geq 2$ points from baseline) and an EASI-75 response (defined as $\geq 75 \%$ improvement from baseline in the score on the EASI) at week 12. Both doses of abrocitinib must have been significantly better than placebo on the basis of both end points in order to have met the goal of the trial.

The three key secondary end points were itch response (defined as $\geq 4$-point improvement from baseline in the score on the PP-NRS) at week 2 and IGA and EASI-75 responses at week 16. Daily scores on the PP-NRS were used in the analysis of this end point rather than average weekly scores.

Additional secondary end points included improvements of at least $50 \%$, at least $90 \%$, and $100 \%$ in the score on the EASI; time to itch response; changes in percentage of body-surfacearea involvement; score on the Patient-Oriented Eczema Measure (POEM; scores range from 0 to 28 , with higher scores indicating greater severity $)^{21}$; score on the Pruritus and Symptoms Assessment for Atopic Dermatitis (PSAAD; scores range from 0 to 10, with higher scores indicating greater severity) ${ }^{22}$; score on the Dermatology Life Quality Index (DLQI; scores range from 0 to 30 , with higher scores indicating greater impairment $)^{23}$; scores on the Hospital Anxiety and Depression Scale (scores range from 0 to 21, with higher scores indicating greater anxiety or depression $)^{24}$; improvements of at least $50 \%$ and at least $75 \%$ in the score on the Scoring of Atopic Dermatitis (SCORAD; scores range from 0 to 103 , with higher scores indicating greater severity $)^{25}$; and change in SCORAD subjective assessments of itch and sleep loss. A PP-NRS score lower than 2 was a post hoc end point. A complete list of the scales used in the trial end-point assessments is provided in Section S4.

At each trial site, the clinical trial investigators, who were unaware of the trial-group assignments, recorded adverse events from the time after a patient signed the informed consent through 28 days after the trial regimen was discontinued. They also recorded abnormal clinical findings and changes from baseline in clinical laboratory values, electrocardiogram measurements, and vital signs from the time after the administration of the first dose of a trial drug or 
placebo through 28 days after the trial regimen was discontinued.

\section{STATISTICAL ANALYSIS}

We determined that a sample size of 700 patients would provide the trial with at least $96 \%$ power to detect a difference of 20 or more percentage points between the abrocitinib dose groups and the placebo group with respect to an IGA response at week 12, assuming that $12 \%$ of the patients in the placebo group would have an IGA response, and with at least $99 \%$ power to detect a between-group difference of 30 or more percentage points with respect to an EASI-75 response at week 12 , assuming that $23 \%$ of the patients in the placebo group would have an EASI-75 response. We used a sequential, Bonferroni-based procedure to control the family-wise type I error rate at 5\% for testing hypotheses across the two primary end points and the three key secondary end points. (Additional details are provided in Section S5 and Fig. S1 in the Supplementary Appendix.)

The two primary end points were tested first for the higher dose of abrocitinib and then for the lower dose at a significance level of 5\%. To meet the primary objective, the differences between the abrocitinib dose groups and the placebo group with respect to the primary end points had to be statistically significant. With regard to the key secondary end points, the significance level was evenly split (2.5\% each) for testing along two hierarchical sequences - sequence A was for the four comparisons of itch response at week 2 (200-mg abrocitinib vs. placebo, 100-mg abrocitinib vs. placebo, 200-mg abrocitinib vs. dupilumab, and 100-mg abroctinib vs. dupilumab), and sequence $B$ was for the four comparisons of an IGA response (200-mg abrocitinib vs. placebo, and 100-mg abrocitinib vs. placebo) and an EASI-75 response (200-mg abrocitinib vs. placebo, and 100-mg abrocitinib vs. placebo) at week 16 (Fig. 2 in the statistical analysis plan, available in the protocol). The analyses of the primary and key secondary end points were based on available data up to and including week 16, with overall family-wise type I error controlled as specified in the statistical analysis plan. If a patient withdrew from the trial and no drug or placebo was dispensed at week 16, all available data for that patient was included in the week-16 analysis. There was no plan for adjustment of confidence intervals of the secondary end points after the key secondary end points, and no definite conclusions can be drawn from these data.

The primary analysis of efficacy was performed in the modified intention-to treat-population, which included all the patients who had undergone randomization and received at least 1 dose of a trial drug or placebo. Primary, key secondary, and all other binary end points were analyzed with the use of two approaches. In the first approach, the Cochran-Mantel-Haenszel statistic, with adjustment for baseline disease severity (moderate or severe), was used to test the hypothesis that there would be no differences between the two trial groups with respect to the primary and key secondary end points; the P values from the Cochran-Mantel-Haenszel test were used to test the hypothesis that there would be no differences between the two trial groups that were compared with respect to the other binary end points. In the second approach, the percentages of patients in each trial group who had responses were reported, and the differences between the two trial groups that were compared were summarized as weighted differences (based on the Cochran-Mantel-Haenszel statistic) and $95 \%$ confidence intervals, calculated with the use of normal approximation.

In the analysis of the end points beyond the three key secondary end points, there was also no plan for the adjustment of confidence intervals for multiple comparisons, and no inferences can be drawn from these data. Sensitivity analyses of the two primary end points are described in Section S6.

Continuous end points were analyzed with a mixed-effect model with repeated measures that used all observed data; the model included trial group, baseline severity, visit, trial-group-byvisit interaction, and relevant baseline values as fixed effects. In the analysis of continuous end points, no imputations were made for missing data because the mixed-effect model with repeated measures yielded valid inferences under the assumption of a missing-at-random mechanism. In the analysis of the binary end points, missing responses for the patients who had permanently withdrawn from the trial were defined as no response with respect to the primary end points at all visits after withdrawal; any observations that were missing intermittently (including 
baseline values) were considered to be missing completely at random and remained as missing in the analysis. Additional details of the analyses are provided in the statistical analysis plan.

\section{RESULTS}

\section{PATIENTS}

A total of 1234 patients underwent screening from October 29, 2018, to August 5, 2019 (the date the last patient was enrolled), and 838 were randomly assigned to a trial group - $226 \mathrm{pa}$ tients were assigned to the 200-mg abrocitinib group, 238 to the 100-mg abrocitinib group, 243 to the dupilumab group, and 131 to the placebo group (Table S1). All but 1 of the 838 patients received a trial drug or placebo. Most patients (91.4\%) completed 16 weeks of treatment (Table S2). The baseline characteristics of the patients, including previous medication use, were similar across groups (Table 1 and Table S3). Details on the use of therapy with a topical medication before and during the trial are provided in Tables S4 through S6. Complete data on the end points of IGA and EASI-75 responses were missing for approximately $10 \%$ of the patients in all groups. Denominators differ at each end point and at each visit because assessments for that end point were missing for the patients who either missed that visit or attended the visit outside the prespecified window.

\section{PRIMARY END POINTS}

In the modified intention-to-treat analysis of efficacy, the first primary end point of an IGA response at week 12 was reported in 106 of 219 patients (48.4\%) in the 200-mg abrocitinib group, 86 of 235 patients $(36.6 \%)$ in the $100-\mathrm{mg}$ abrocitinib group, 88 of 241 patients $(36.5 \%)$ in the dupilumab group, and 18 of 129 patients (14.0\%) in the placebo group (Table 2). The second primary outcome of an EASI-75 response at week 12 was reported in 154 of 219 patients (70.3\%) in the 200-mg abrocitinib group, 138 of 235 patients $(58.7 \%)$ in the 100 -mg abrocitinib group, 140 of 241 patients $(58.1 \%)$ in the dupilumab group, and 35 of 129 patients (27.1\%) in the placebo group.

The weighted difference in the percentage of patients who had an IGA response at week 12 between the 200-mg abrocitinib group and the placebo group was 34.8 percentage points $(95 \%$
CI, 26.1 to 43.5 ; $\mathrm{P}<0.001$ ) and that between the 100-mg abrocitinib group and the placebo group was 23.1 percentage points (95\% CI, 14.7 to 31.4; $\mathrm{P}<0.001)$. The weighted difference in the percentage of patients who had an EASI-75 response at week 12 between the 200-mg abrocitinib group and the placebo group was 43.2 percentage points (95\% CI, 33.7 to 52.7$)$ and that between the 100-mg abrocitinib group and the placebo group was 31.9 percentage points $(95 \%$ CI, 22.2 to 41.6) ( $\mathrm{P}<0.001$ for both comparisons). The results of sensitivity and additional efficacy analyses are provided in Tables S7 to S13.

\section{KEY SECONDARY END POINTS}

An itch response at week 2 (the first key secondary end point) occurred in 111 of 226 patients (49.1\%) in the 200-mg abrocitinib group, 75 of 236 patients (31.8\%) in the 100-mg abrocitinib group, 63 of 239 patients (26.4\%) in the dupilumab group, and 18 of 130 patients (13.8\%) in the placebo group. The weighted difference in the percentage of patients who had an itch response at week 2 between the 200-mg abrocitinib group and the placebo group was 34.9 percentage points (95\% CI, 26.0 to 43.7 ) and that between the 100-mg abrocitinib group and the placebo group was 17.9 percentage points $(95 \% \mathrm{CI}, 9.5$ to 26.3) ( $\mathrm{P}<0.001$ for both comparisons). The weighted difference in the percentage of patients who had an itch response at week 2 between the 200-mg abrocitinib group and the dupilumab group was 22.1 percentage points favoring this dose of abrocitinib (95\% CI, 13.5 to 30.7; $\mathrm{P}<0.001)$. The weighted difference between the 100-mg abrocitinib group and the dupilumab group was 5.2 percentage points $(95 \% \mathrm{CI},-2.9$ to $13.4 ; \mathrm{P}=0.20$ ), indicating no significant difference between the trial groups for this dose of abrocitinib. The median time to itch response in each trial group is provided in Figure 1.

An IGA response at week 16 (the second key secondary end point) occurred in 105 of 221 patients (47.5\%) in the 200-mg abrocitinib group, 80 of 230 patients (34.8\%) in the 100 -mg abrocitinib group, 90 of 232 patients $(38.8 \%)$ in the dupilumab group, and 16 of 124 patients (12.9\%) in the placebo group. The weighted difference in the percentage of patients who had an IGA response at week 16 between the 200-mg abrocitinib group and the placebo group was 35.0 percentage points (95\% CI, 26.3 to 43.7 ) and that 
Table 1. Demographic and Disease Characteristics of the Patients at Baseline.*

\begin{tabular}{|c|c|c|c|c|c|}
\hline Characteristic & $\begin{array}{c}\text { Total } \\
(\mathrm{N}=837)\end{array}$ & $\begin{array}{c}\text { Abrocitinib, } \\
200 \text { mg Once Daily } \\
(N=226)\end{array}$ & $\begin{array}{l}\text { Abrocitinib, } \\
100 \text { mg Once Daily } \\
\text { (N=238) }\end{array}$ & $\begin{array}{c}\text { Dupilumab, } \\
300 \text { mg Every } \\
\text { Other Week } \\
(\mathrm{N}=242)\end{array}$ & $\begin{array}{l}\text { Placebo } \\
(\mathrm{N}=131)\end{array}$ \\
\hline Age $-y r$ & $37.7 \pm 14.7$ & $38.8 \pm 14.5$ & $37.3 \pm 14.8$ & $37.1 \pm 14.6$ & $37.4 \pm 15.2$ \\
\hline Female sex — no. (\%) & $428(51.1)$ & $122(54.0)$ & $118(49.6)$ & $134(55.4)$ & $54(41.2)$ \\
\hline \multicolumn{6}{|l|}{ Race - no. (\%)† } \\
\hline White & $606(72.4)$ & $161(71.2)$ & $182(76.5)$ & $176(72.7)$ & $87(66.4)$ \\
\hline Black & $35(4.2)$ & $9(4.0)$ & $6(2.5)$ & $14(5.8)$ & $6(4.6)$ \\
\hline Asian & $178(21.3)$ & $53(23.5)$ & $48(20.2)$ & $46(19.0)$ & $31(23.7)$ \\
\hline Other & $18(2.2)$ & $3(1.3)$ & $2(0.8)$ & $6(2.5)$ & $7(5.3)$ \\
\hline Duration of atopic dermatitis $-y r$ & $22.7 \pm 15.4$ & $23.4 \pm 15.6$ & $22.7 \pm 16.3$ & $22.8 \pm 14.8$ & $21.4 \pm 14.4$ \\
\hline \multicolumn{6}{|l|}{ IGA score - no. (\%) } \\
\hline 0, clear & 0 & 0 & 0 & 0 & 0 \\
\hline 1 , almost clear & 0 & 0 & 0 & 0 & 0 \\
\hline 2, mild & 0 & 0 & 0 & 0 & 0 \\
\hline 3 , moderate & $541(64.6)$ & $138(61.1)$ & $153(64.3)$ & $162(66.9)$ & $88(67.2)$ \\
\hline 4 , severe & $296(35.4)$ & $88(38.9)$ & $85(35.7)$ & $80(33.1)$ & $43(32.8)$ \\
\hline EASI score & $30.9 \pm 12.8$ & $32.1 \pm 13.1$ & $30.3 \pm 13.5$ & $30.4 \pm 12.0$ & $31.0 \pm 12.6$ \\
\hline Body-surface-area involvement — \% & $48.5 \pm 23.1$ & $50.8 \pm 23.0$ & $48.1 \pm 23.1$ & $46.5 \pm 22.1$ & $48.9 \pm 24.9$ \\
\hline PP-NRS scored & $7.3 \pm 1.7$ & $7.6 \pm 1.5$ & $7.1 \pm 1.7$ & $7.3 \pm 1.7$ & $7.1 \pm 1.8$ \\
\hline SCORAD score & $67.9 \pm 12.6$ & $69.3 \pm 12.7$ & $66.8 \pm 13.8$ & $67.9 \pm 11.4$ & $67.9 \pm 12.0$ \\
\hline POEM score $\|$ & $21.1 \pm 5.5$ & $21.5 \pm 5.3$ & $20.9 \pm 5.5$ & $21.2 \pm 5.5$ & $20.4 \pm 6.1$ \\
\hline DLQI score ${ }^{* * *}$ & $15.7 \pm 6.6$ & $16.3 \pm 6.6$ & $15.5 \pm 6.4$ & $15.6 \pm 6.7$ & $15.2 \pm 6.9$ \\
\hline \multicolumn{6}{|l|}{ Coexisting medical conditions - no. (\%) } \\
\hline Asthma & $284(33.9)$ & $82(36.3)$ & $79(33.2)$ & $75(31.0)$ & $48(36.6)$ \\
\hline Allergic conjunctivitis & $79(9.4)$ & $18(8.0)$ & $21(8.8)$ & $26(10.7)$ & $14(10.7)$ \\
\hline Food allergy & $125(14.9)$ & $39(17.3)$ & $36(15.1)$ & $36(14.9)$ & $14(10.7)$ \\
\hline
\end{tabular}

* Plus-minus values are means \pm SD. Percentages may not total 100 because of rounding. IGA denotes Investigator's Global Assessment.

$\dagger$ Race was reported by the patients.

7 Scores on the Eczema Area and Severity Index (EASI) range from 0 to 72, with higher scores indicating more severe disease.

$\int$ Scores on the Peak Pruritus Numerical Rating Scale (PP-NRS) represent maximum itch severity in the previous 24 hours and range from 0 to 10, with higher scores representing more severe itch. A change in score of at least 4 points represents clinically meaningful improvement.

9 The Scoring of Atopic Dermatitis (SCORAD) combines investigator-assessed disease severity, body-surface-area involvement, and patientreported itch and sleep. Scores range from 0 to 103, with higher scores representing more severe disease. A change in score of 8.7 points has been estimated as the minimal clinically important difference.

|| Scores on the Patient-Oriented Eczema Measure (POEM) represent frequency of symptoms and range from 0 to 28 , with higher scores indicating greater severity of atopic dermatitis.

*** The Dermatology Life Quality Index (DLQI) is used to estimate disease-specific, health-related quality of life. Scores range from 0 to 30 , with higher scores indicating greater impairment. A score of 0 or 1 represents no effect on health-related quality of life. A change in score of 4 points has been estimated as the minimal clinically important difference.

between the 100-mg abrocitinib group and the placebo group was 22.1 percentage points $(95 \%$ CI, 13.7 to 30.5) ( $\mathrm{P}<0.001$ for both comparisons). The weighted difference in the percentage of patients who had an IGA response at week 16 between the 200-mg abrocitinib group and the dupilumab group was 9.4 percentage points (95\% CI, 0.4 to 18.5), and the difference between the 100-mg abrocitinib group and the dupilumab group was -3.5 percentage points $(95 \% \mathrm{CI},-12.2$ to 5.2).

An EASI-75 response at week 16 (the third key 


\begin{tabular}{|c|c|c|c|c|}
\hline End Point & $\begin{array}{c}\text { Abrocitinib, } \\
200 \text { mg Once Daily } \\
(N=226)\end{array}$ & $\begin{array}{l}\text { Abrocitinib, } \\
100 \text { mg Once Daily } \\
\text { (N=238) }\end{array}$ & $\begin{array}{l}\text { Dupilumab, } \\
300 \text { mg Every } \\
\text { Other Week } \\
(\mathrm{N}=242) \dagger\end{array}$ & $\begin{array}{l}\text { Placebo } \\
(\mathrm{N}=131)\end{array}$ \\
\hline \multicolumn{5}{|l|}{ Primary end points } \\
\hline IGA response at week $12-$ no./total no. (\%) $\ddagger$ & $106 / 219(48.4)$ & $86 / 235(36.6)$ & $88 / 241(36.5)$ & $18 / 129(14.0)$ \\
\hline $\begin{array}{l}\text { Difference from placebo }(95 \% \mathrm{Cl}) \text { - percentage } \\
\text { points }\end{array}$ & $34.8(26.1$ to 43.5$)$ & 23.1 (14.7 to 31.4$)$ & 22.5 (14.2 to 30.9$)$ & NA \\
\hline$P$ value & $<0.001$ & $<0.001$ & & \\
\hline EASI-75 response at week $12-$ no./total no. (\%) $\int$ & $154 / 219(70.3)$ & $138 / 235(58.7)$ & $140 / 241(58.1)$ & $35 / 129(27.1)$ \\
\hline $\begin{array}{l}\text { Difference from placebo }(95 \% \mathrm{Cl}) \text { - percentage } \\
\text { points }\end{array}$ & $43.2(33.7$ to 52.7$)$ & 31.9 (22.2 to 41.6$)$ & $30.9(21.2$ to 40.6$)$ & NA \\
\hline$P$ value & $<0.001$ & $<0.001$ & & \\
\hline \multicolumn{5}{|l|}{ Key secondary end points } \\
\hline Itch response at week $2-$ no./total no. (\%) 9 & $111 / 226(49.1)$ & $75 / 236(31.8)$ & $63 / 239(26.4)$ & $18 / 130(13.8)$ \\
\hline $\begin{array}{l}\text { Difference from placebo }(95 \% \mathrm{Cl}) \text { - percentage } \\
\text { points }\end{array}$ & 34.9 (26.0 to 43.7$)$ & 17.9 (9.5 to 26.3$)$ & 12.5 (4.4 to 20.7$)$ & NA \\
\hline$P$ value & $<0.001$ & $<0.001$ & & \\
\hline $\begin{array}{l}\text { Difference from dupilumab }(95 \% \mathrm{Cl}) \text { - percentage } \\
\text { points }\end{array}$ & 22.1 (13.5 to 30.7$)$ & $5.2(-2.9$ to 13.4$)$ & NA & NA \\
\hline$P$ value & $<0.001$ & $0.20 \|$ & & \\
\hline IGA response at week $16-$ no./total no. (\%) & $105 / 221(47.5)$ & $80 / 230(34.8)$ & $90 / 232(38.8)$ & $16 / 124(12.9)$ \\
\hline $\begin{array}{l}\text { Difference from placebo }(95 \% \mathrm{Cl}) \text { - percentage } \\
\text { points }\end{array}$ & $35.0(26.3$ to 43.7$)$ & 22.1 (13.7 to 30.5$)$ & 25.6 (17.1 to 34.1$)$ & NA \\
\hline$P$ value & $<0.001$ & $<0.001$ & & \\
\hline $\begin{array}{l}\text { Difference from dupilumab }(95 \% \mathrm{Cl}) \text { - percentage } \\
\text { points }\end{array}$ & 9.4 (0.4 to 18.5$)$ & $-3.5(-12.2$ to 5.2$)$ & NA & NA \\
\hline EASI-75 response at week $16-$ no./total no. (\%) & $157 / 221(71.0)$ & $138 / 229(60.3)$ & $152 / 232(65.5)$ & $38 / 124(30.6)$ \\
\hline $\begin{array}{l}\text { Difference from placebo }(95 \% \mathrm{Cl}) \text { - percentage } \\
\text { points }\end{array}$ & $40.4(30.4$ to 50.4$)$ & 29.7 (19.5 to 39.9$)$ & 34.7 (24.6 to 44.8$)$ & NA \\
\hline$P$ value & $<0.001$ & $<0.001$ & & \\
\hline Difference from dupilumab $(95 \% \mathrm{CI})$ & $5.5(-3.1$ to 14.1$)$ & $-5.1(-13.9$ to 3.7$)$ & NA & NA \\
\hline \multicolumn{5}{|c|}{$\begin{array}{l}\text { Denominators may vary owing to intermittent missing values. NA denotes not applicable. } \\
\text { Comparisons between the dupilumab group and other trial groups were not multiplicity-controlled, except with respect to itch response at } \\
\text { week } 2 \text {. } \\
\text { IGA response was defined as a score of } 0 \text { (clear) or } 1 \text { (almost clear), with at least a } 2 \text {-point improvement from baseline. } \\
\text { EASI-75 response was defined as an improvement of at least } 75 \% \text { from baseline in the score. } \\
\text { Itch response was defined as an improvement of at least } 4 \text { points from baseline in the score on the PP-NRS. } \\
\text { Hierarchal testing for comparisons between the abrocitinib groups and the dupilumab group failed at this point, so subsequent P values a } \\
\text { not reported. }\end{array}$} \\
\hline
\end{tabular}

secondary end point) occurred in 157 of 221 patients $(71.0 \%)$ in the $200-\mathrm{mg}$ abrocitinib group, 138 of 229 patients (60.3\%) in the $100-\mathrm{mg}$ abrocitinib group, 152 of 232 patients (65.5\%) in the dupilumab group, and 38 of 124 patients $(30.6 \%)$ in the placebo group. The weighted difference in the percentage of patients who had an EASI-75 response at week 16 between the 200-mg abrocitinib group and the placebo group was
40.4 percentage points (95\% CI, 30.4 to 50.4 ; $\mathrm{P}<0.001$ ) and that between the 100 -mg abrocitinib group and the placebo group was 29.7 percentage points (95\% CI, 19.5 to 39.9; $\mathrm{P}<0.001$ ). The weighted difference in the percentage of patients who had an EASI-75 response at week 16 between the 200-mg abrocitinib group and the dupilumab group was 5.5 percentage points (95\% CI, -3.1 to 14.1 ), and the difference be- 


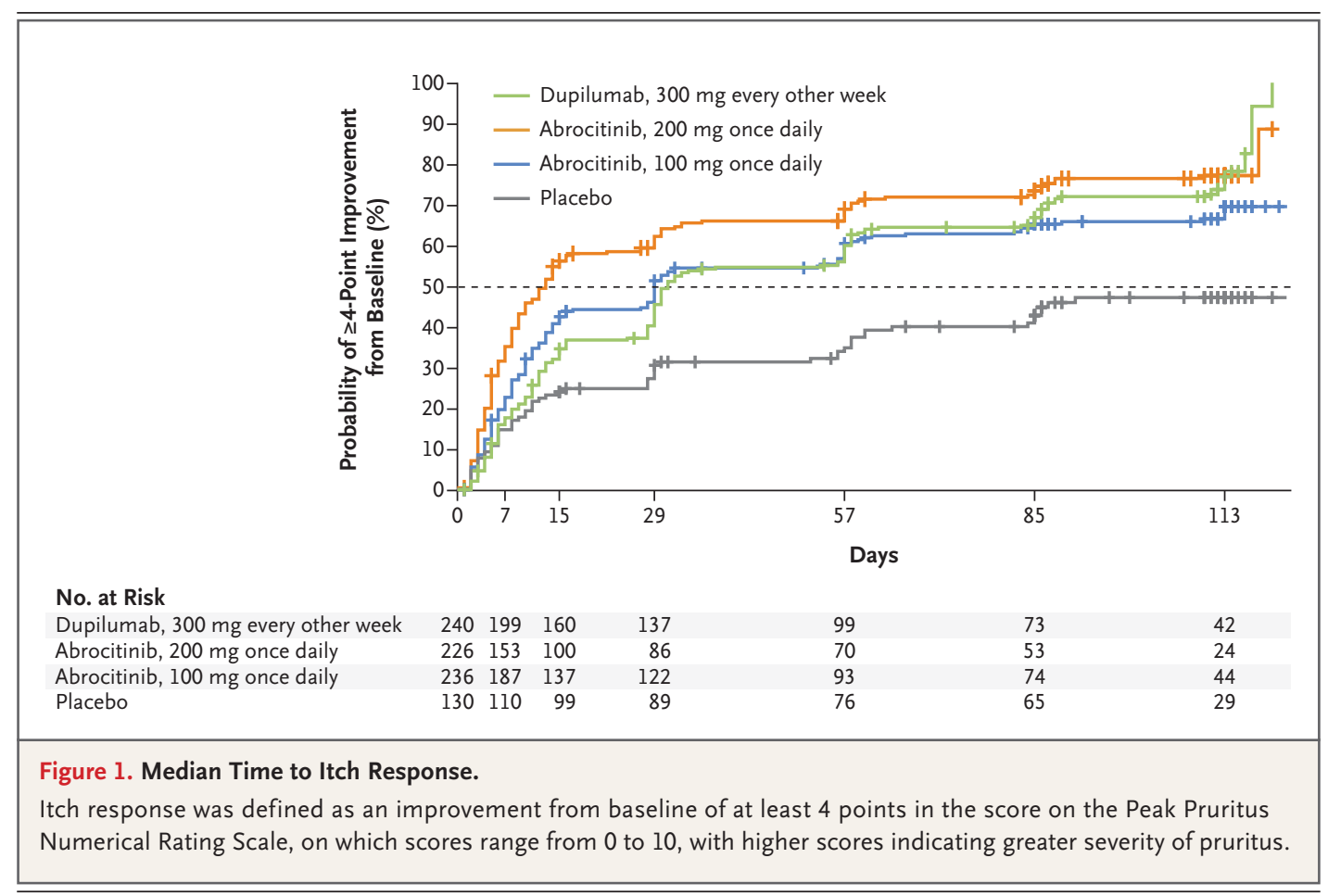

tween the 100-mg group and the dupilumab group was -5.1 percentage points $(95 \% \mathrm{CI},-13.9$ to 3.7).

Therefore, both doses of abrocitinib were not significantly different from dupilumab with respect to an EASI-75 response at week 16. The weighted difference in the percentage of patients who had an IGA response at week 16 between the 200-mg abrocitinib group and the dupilumab group was in the same direction as that at week 12; however, the absence of a prespecified plan for adjustment of confidence intervals for multiple comparisons precludes conclusions from this result.

The percentages of patients who had an IGA response and of those who had an EASI-75 response over time are provided in Figure 2. Additional secondary and post hoc efficacy end points are provided in Tables S14 through S18 and Figures S2 through S5. These end points were not adjusted for multiplicity, and no clinical inferences can be made from these data.

\section{SAFETY}

No deaths, major cardiovascular adverse events, or thromboembolic events occurred during the trial. Two malignant neoplasms (confirmed by the external data monitoring committee) were reported - one in the 200-mg abrocitinib group (cutaneous squamous-cell carcinoma) and one in the dupilumab group (invasive intraductal breast neoplasia). The incidence of adverse events that occurred during the treatment period (defined as any adverse event that occurred after the administration of the first dose of a trial drug or placebo through 28 days after the trial regimen was discontinued) was higher in the 200-mg abrocitinib group than in the other groups (Table 3). The percentages of patients who had serious or severe adverse events during the treatment period or adverse events that led to discontinuation of the trial regimen were similar across the trial groups.

A total of six adverse events during the treatment period were reported by at least $5 \%$ of patients in any trial group (Table 3). Nausea was reported more frequently in the abrocitinib groups (25 patients [11.1\%] who received the 200-mg dose and 10 [4.2\%] who received the $100-\mathrm{mg}$ dose) than in the dupilumab group (7 [2.9\%]) or the placebo group (2 $[1.5 \%]$ ). The trial regimen was discontinued because of nausea in 1 patient in the 200-mg abrocitinib group, 1 patient in the 100-mg abrocitinib group, and 1 patient in the 


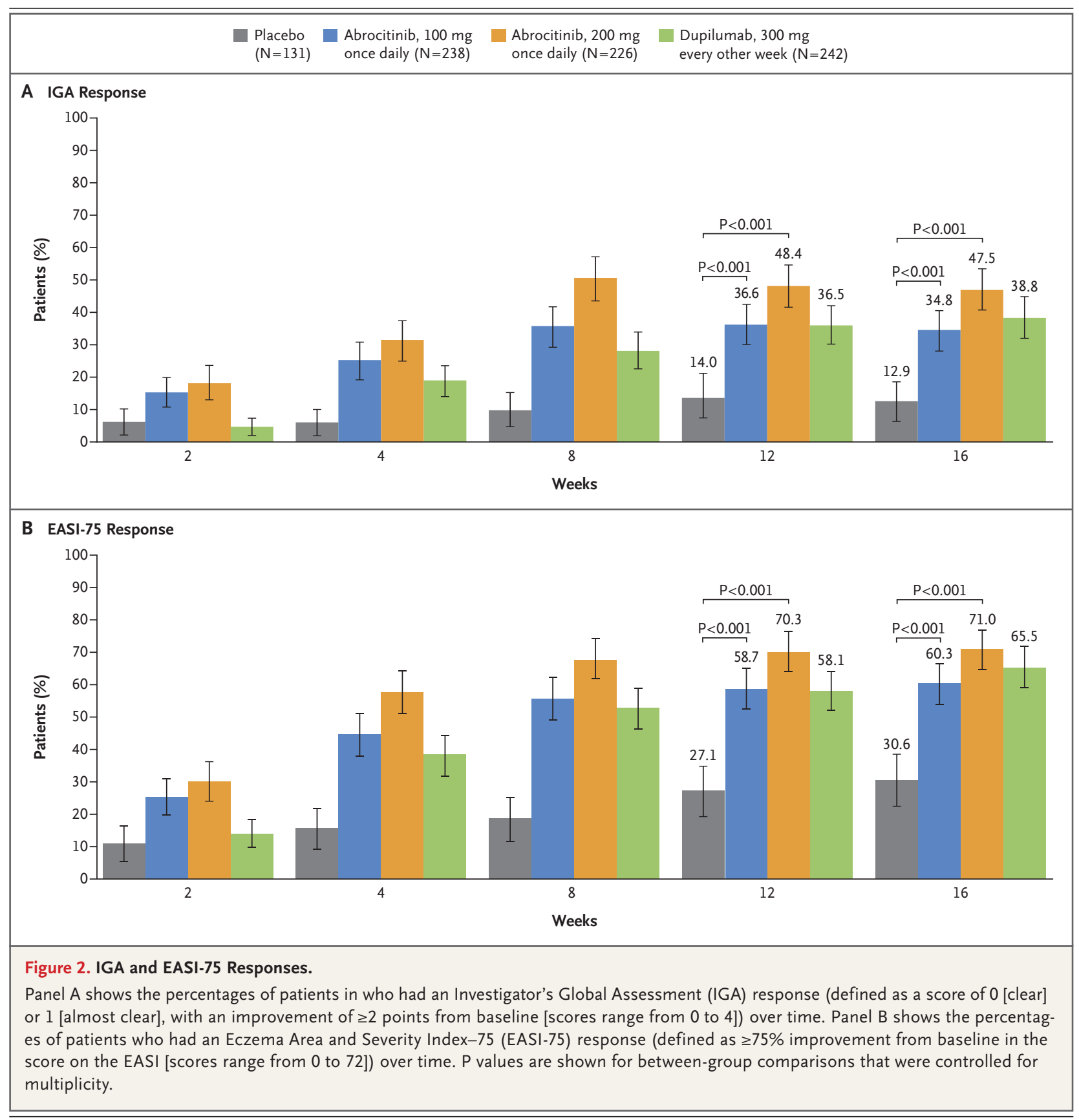

dupilumab group. Acne, which was mild or moderate in all cases, was reported more frequently in the abrocitinib groups (15 patients [6.6\%] who received the 200-mg dose and 7 [2.9\%] who received the 100-mg dose) than in the dupilumab group (3 $[1.2 \%])$ or the placebo group ( $0 \mathrm{pa}-$ tients). Conjunctivitis was reported more frequently in the dupilumab group (15 patients [6.2\%]) than in the abrocitinib groups (3 [1.3\%] who received the 200-mg dose and 22 [0.8\%] who received the 100-mg dose) or the placebo group (3 [2.3\%]). The percentages of patients who had nasopharyngitis, upper respiratory tract infection, or headache were similar in the trial groups (Table 3).

Three serious infections were reported in $2 \mathrm{pa}-$ 


\begin{tabular}{|c|c|c|c|c|}
\hline Event & $\begin{array}{c}\text { Abrocitinib, } \\
200 \text { mg Once Daily } \\
(N=226)\end{array}$ & $\begin{array}{l}\text { Abrocitinib, } \\
100 \text { mg Once Daily } \\
(\mathrm{N}=238) \\
\text { number of patients }\end{array}$ & $\begin{array}{l}\text { Dupilumab, } \\
300 \text { mg Every Other } \\
\text { Week } \\
(\mathrm{N}=242) \\
\text { vith event (percent) }\end{array}$ & $\begin{array}{r}\text { Placebo } \\
(\mathrm{N}=131)\end{array}$ \\
\hline$\geq 1$ Adverse event & $140(61.9)$ & $121(50.8)$ & $121(50.0)$ & $70(53.4)$ \\
\hline Serious adverse event* & $2(0.9)$ & $6(2.5)$ & $2(0.8)$ & $5(3.8)$ \\
\hline Severe adverse event* & $4(1.8)$ & $5(2.1)$ & $2(0.8)$ & $3(2.3)$ \\
\hline Adverse event leading to study discontinuation & $10(4.4)$ & $6(2.5)$ & $8(3.3)$ & $5(3.8)$ \\
\hline \multicolumn{5}{|l|}{$\begin{array}{l}\text { Adverse event reported in } \geq 5 \% \text { of patients in } \\
\text { any group }\end{array}$} \\
\hline Nausea & $25(11.1)$ & $10(4.2)$ & $7(2.9)$ & $2(1.5)$ \\
\hline Conjunctivitis & $3(1.3)$ & $2(0.8)$ & $15(6.2)$ & $3(2.3)$ \\
\hline Nasopharyngitis & $15(6.6)$ & $22(9.2)$ & $23(9.5)$ & $9(6.9)$ \\
\hline Upper respiratory tract infection & $9(4.0)$ & $12(5.0)$ & $9(3.7)$ & $6(4.6)$ \\
\hline Headache & $15(6.6)$ & $10(4.2)$ & $13(5.4)$ & $6(4.6)$ \\
\hline Acne & $15(6.6)$ & $7(2.9)$ & $3(1.2)$ & 0 \\
\hline Herpes zoster'† & $4(1.8)$ & $2(0.8)$ & 0 & 0 \\
\hline Thrombocytopenia† & $2(0.9)$ & 0 & 0 & 0 \\
\hline
\end{tabular}

* Serious and severe adverse events are defined in the protocol.

$\dagger$ Herpes zoster and thrombocytopenia were adverse events that did not reach the threshold of at least $5 \%$ in any group but have been included owing to clinical interest in the incidence of these events with the use of JAK inhibitors.

tients $(0.8 \%)$ in the $100-\mathrm{mg}$ abrocitinib group (pneumonia and oral herpes [herpes labialis] in 1 patient [who was withdrawn from the trial because of pneumonia] and infectious diarrhea in 1 patient) (Table S19); all the cases resolved. A total of 4 patients $(1.8 \%)$ in the $200-\mathrm{mg}$ abrocitinib group and $2(0.8 \%)$ in the $100-\mathrm{mg}$ group had herpes zoster infection. In the 200-mg dose group, severity was mild in 3 patients and moderate in 1 patient; in the 100-mg dose group, severity was moderate in 1 patient and severe in 1 patient. All the cases of herpes zoster infection were uncomplicated and did not involve dose changes or withdrawal from the trial, and none were multidermatomal; all the cases resolved except for one that was resolving at the time of this report. Eczema herpeticum was reported in 1 patient in the 100-mg abrocitinib group and 1 patient in the placebo group.

Dose-dependent decreases in the median platelet counts were reported among the patients who received abrocitinib, but no patient had a platelet count of less than 75,000 per cubic millimeter, as confirmed by means of repeated blood measurements (Table S21). Thrombocytopenia was reported in 2 patients (0.9\%) in the $200-\mathrm{mg}$ abrocitinib group, with a nadir of platelet counts of 110,000 per cubic millimeter in one patient and 118,000 per cubic millimeter in the other. The patients who received abrocitinib had dosedependent increases in the median creatine kinase level (without rhabdomyolysis), the mean total cholesterol level, the mean low-density lipoprotein (LDL) cholesterol level, and the mean high-density lipoprotein (HDL) cholesterol level, but the mean LDL:HDL cholesterol ratio remained unchanged (Tables S20 through S28). Adverse events after week 16 are reported in Table S29.

\section{DISCUSSION}

In the JADE COMPARE trial, abrocitinib at a dose of either $200 \mathrm{mg}$ or $100 \mathrm{mg}$ once daily resulted in significantly greater reductions in signs and symptoms of moderate-to-severe atopic dermatitis than placebo on the basis of IGA and EASI-75 responses at weeks 12 and 16. The 100-mg 
dose of abrocitinib was not significantly different from dupilumab with respect to the three key secondary end points of the trial. The 200-mg dose of abrocitinib was superior to dupilumab with respect to itch response at week 2 but not to an EASI-75 response at week 16; no conclusion could be drawn regarding the difference between the 200-mg dose of abrocitinib and dupilumab with respect to an IGA response. In contrast to previous clinical trials that evaluated abrocitinib as monotherapy in patients with atopic dermatitis, in the current trial, we evaluated abrocitinib in patients with atopic dermatitis who were receiving background therapy with topical medications..$^{6-8}$

Adverse events occurred in a higher percentage of patients in the 200-mg abrocitinib group than in the placebo group or the dupilumab group; the percentages of patients who had adverse events in the 100-mg abrocitinib group were similar to percentages observed among those in the dupilumab group. The main adverse events with abrocitinib were nausea, acne, nasopharyngitis, and headache. Conjunctivitis occurred more frequently with dupilumab than with placebo, as has been reported in previous trials, ${ }^{26}$ whereas the percentage of patients who had conjunctivitis in either abrocitinib group was similar to that in the placebo group. Serious and opportunistic infections are considered to be a risk with JAK inhibitors in patients with rheumatoid arthritis. ${ }^{27}$ In the current trial, herpes zoster was reported more frequently with abrocitinib than with placebo or dupilumab, and serious infections occurred in two patients receiving abrocitinib.

This trial has several limitations. In terms of atopic dermatitis as a lifelong disease, this 16week trial did not establish the long-term efficacy and safety of abrocitinib. The trial was not formally designed to evaluate the superiority of abrocitinib over dupilumab with respect to the two primary end points. Clinical inferences could not be drawn from the between-group differences with respect to the secondary end points beyond the three key secondary end points because of the lack of a plan for adjustment of confidence intervals for multiple comparisons. With respect to the binary end points, the handling of missing responses for patients who permanently withdrew from the trial (who were designated as having a nonresponse after withdrawal) may have introduced bias against placebo. Reported acne reactions were not detailed enough to confirm the presence or absence of comedonal acne. Finally, this trial involved only adults.

In this trial involving patients with atopic dermatitis who were receiving background topical therapy, those who received abrocitinib at either the 200-mg or 100-mg dose had greater reductions in signs and symptoms of atopic dermatitis at 12 weeks than those who received placebo. The 200-mg dose, but not the 100-mg dose, of abrocitinib was superior to dupilumab with respect to itch response at week 2 , but neither abrocitinib dose differed significantly from dupilumab with respect to most other key secondary end points at week 16. Longer and larger trials are necessary to determine the efficacy and safety of abrocitinib and to compare it with other JAK inhibitors and with biologic agents used for the treatment of atopic dermatitis.

\section{Supported by Pfizer.}

Disclosure forms provided by the authors are available with the full text of this article at NEJM.org.

A data sharing statement provided by the authors is available with the full text of this article at NEJM.org.

We thank Robert J. Schoen, Pharm.D., and Jennifer C. Jaworski, M.S., at ApotheCom, for medical writing and editorial support with earlier versions of the manuscript after the first draft.

The authors' full names and academic degrees are as follows: Thomas Bieber, M.D., Ph.D., Eric L. Simpson, M.D., Jonathan I. Silverberg, M.D., Ph.D., M.P.H., Diamant Thaçi, M.D., Carle Paul, M.D., Ph.D., Andrew E. Pink, Ph.D., Yoko Kataoka, M.D., Chia-Yu Chu, M.D., Ph.D., Marco DiBonaventura, Ph.D., Ricardo Rojo, M.D., Jeremias Antinew, M.D., Ileana Ionita, Ph.D., Rodney Sinclair, M.D., Seth Forman, M.D., Jacek Zdybski, M.D., Pinaki Biswas, Ph.D., Bimal Malhotra, Ph.D., Fan Zhang, Ph.D., and Hernan Valdez, M.D.

The authors' affiliations are as follows: the University Hospital of Bonn, Bonn (T.B.), and University of Lübeck, Lübeck (D.T.) — both in Germany; Oregon Health and Science University, Portland (E.L.S.); George Washington University School of Medicine and Health Sciences, Washington, DC (J.I.S.); Toulouse University and Centre Hospitalier Universitaire, Toulouse, France (C.P.); St. John's Institute of Dermatology, Guy's and St. Thomas' NHS Foundation Trust, London (A.E.P.); Osaka Habikino Medical Center, Osaka, Japan (Y.K.); the Department of Dermatology, National Taiwan University Hospital and National Taiwan University College of Medicine, Taipei (C.-Y.C.); Pfizer, New York (M.D., P.B., B.M., H.V.); Pfizer, Groton, CT (R.R., J.A., I.I., F.Z.); Sinclair Dermatology, East Melbourne, VIC, Australia (R.S.); ForCare Clinical Research, Tampa, FL (S.F.); and Dermedic Jacek Zdybski, Ostrowiec Świętokrzyski, Poland (J.Z.). 
REFERENCES

1. Eckert L, Gupta S, Amand C, Gadkari A, Mahajan P, Gelfand JM. The burden of atopic dermatitis in US adults: health care resource utilization data from the 2013 National Health and Wellness Survey. J Am Acad Dermatol 2018;78(1):54-61.e1.

2. Silverberg JI, Gelfand JM, Margolis DJ, et al. Patient burden and quality of life in atopic dermatitis in US adults: a population-based cross-sectional study. Ann Allergy Asthma Immunol 2018;121:340-7. 3. Boguniewicz M, Fonacier L, GuttmanYassky E, Ong PY, Silverberg J, Rosen Farrar J. Atopic dermatitis yardstick: practical recommendations for an evolving therapeutic landscape. Ann Allergy Asthma Immunol 2018;120(1):10-22.e2.

4. Guttman-Yassky E, Silverberg JI, Nemoto O, et al. Baricitinib in adult patients with moderate-to-severe atopic dermatitis: a phase 2 parallel, double-blinded, randomized placebo-controlled multipledose study. J Am Acad Dermatol 2019; 80(4):913-21.e9.

5. Guttman-Yassky E, Thaçi D, Pangan $\mathrm{AL}$, et al. Upadacitinib in adults with moderate to severe atopic dermatitis: 16week results from a randomized, placebocontrolled trial. J Allergy Clin Immunol 2020;145:877-84.

6. Gooderham MJ, Forman SB, Bissonnette $\mathrm{R}$, et al. Efficacy and safety of oral Janus kinase 1 inhibitor abrocitinib for patients with atopic dermatitis: a phase 2 randomized clinical trial. JAMA Dermatol 2019;155:1371-9.

7. Simpson EL, Sinclair R, Forman S, et al. Efficacy and safety of abrocitinib in adults and adolescents with moderate-to-severe atopic dermatitis: results from JADE MONO-1, a randomised, phase 3 clinical trial. Lancet 2020;396:255-66.

8. Silverberg JI, Simpson EL, Thyssen JP, et al. Efficacy and safety of abrocitinib in patients with moderate-to-severe atopic dermatitis: a randomized phase 3 clinical trial. JAMA Dermatol 2020;156:863-73.

9. Center for Drug Evaluation and Research. Dupixent (dupilumab) clinical re- view. 2016 (https://www.accessdata.fda.gov/ drugsatfda_docs/nda/2017/761055Orig1s 000MedR.pdf)

10. Blauvelt A, Simpson EL, Tyring SK, et al Dupilumab does not affect correlates of vaccine-induced immunity: a randomized placebo-controlled trial in adults with moderate-to-severe atopic dermatitis. J Am Acad Dermatol 2019;80(1):158-167.e1.

11. Guttman-Yassky E, Bissonnette R, Ungar B, et al. Dupilumab progressively improves systemic and cutaneous abnormalities in patients with atopic dermatitis. J Allergy Clin Immunol 2019;143:15572 .

12. de Bruin-Weller $M$, Thaçi D, Smith $\mathrm{CH}$, et al. Dupilumab with concomitan topical corticosteroid treatment in adults with atopic dermatitis with an inadequate response or intolerance to ciclosporin $\mathrm{A}$ or when this treatment is medically inadvisable: a placebo-controlled, randomized phase III clinical trial (LIBERTY AD CAFÉ). Br J Dermatol 2018;178:1083-101.

13. Simpson EL, Bieber T, Guttman-Yassky $\mathrm{E}$, et al. Two phase 3 trials of dupilumab versus placebo in atopic dermatitis. N Engl J Med 2016;375:2335-48.

14. Beck LA, Thaçi D, Hamilton JD, et al Dupilumab treatment in adults with moderate-to-severe atopic dermatitis. $\mathrm{N}$ Engl Med 2014;371:130-9.

15. Pavel AB, Zhou L, Diaz A, et al. The proteomic skin profile of moderate-tosevere atopic dermatitis patients shows an inflammatory signature. J Am Acad Dermatol 2020;82:690-9.

16. Leach MW, Rottman JB, Hock MB Finco D, Rojko JL, Beyer JC. Immunogenicity/hypersensitivity of biologics. Toxicol Pathol 2014;42:293-300.

17. Strand V, Balsa A, Al-Saleh J, et al. Immunogenicity of biologics in chronic inflammatory diseases: a systematic review. BioDrugs 2017;31:299-316.

18. Futamura M, Leshem YA, Thomas KS Nankervis H, Williams HC, Simpson EL. A systematic review of Investigator Global Assessment (IGA) in atopic dermatitis
(AD) trials: many options, no standards. J Am Acad Dermatol 2016;74:288-94.

19. Hanifin JM, Thurston M, Omoto M, Cherill R, Tofte SJ, Graeber M. The Eczema Area and Severity Index (EASI): assessment of reliability in atopic dermatitis. Exp Dermatol 2001;10:11-8.

20. Phan NQ, Blome C, Fritz F, et al. Assessment of pruritus intensity: prospective study on validity and reliability of the visual analogue scale, numerical rating scale and verbal rating scale in 471 patients with chronic pruritus. Acta Derm Venereol 2012;92:502-7.

21. Charman CR, Venn AJ, Williams HC. The patient-oriented eczema measure: development and initial validation of a new tool for measuring atopic eczema severity from the patients' perspective. Arch Dermatol 2004;140:1513-9.

22. Hall R, Lebwohl MG, Bushmakin AG, et al. Development and content validation of Pruritus and Symptoms Assessment for Atopic Dermatitis (PSAAD) in adolescents and adults with moderate-to-severe AD. Dermatol Ther (Heidelb) 2021;11:221-33. 23. Finlay AY, Khan GK. Dermatology Life Quality Index (DLQI) - a simple practical measure for routine clinical use. Clin Exp Dermatol 1994;19:210-6.

24. Zigmond AS, Snaith RP. The Hospital Anxiety and Depression Scale. Acta Psychiatr Scand 1983;67:361-70.

25. European Task Force on Atopic Dermatitis. Severity scoring of atopic dermatitis: the SCORAD index - consensus report of the European Task Force on Atopic Dermatitis. Dermatology 1993;186: 23-31.

26. Akinlade B, Guttman-Yassky E, de Bruin-Weller $M$, et al. Conjunctivitis in dupilumab clinical trials. Br J Dermatol 2019;181:459-73.

27. Bechman K, Subesinghe S, Norton S, et al. A systematic review and meta-analysis of infection risk with small molecule JAK inhibitors in rheumatoid arthritis. Rheumatology (Oxford) 2019;58:1755-66. Copyright (c) 2021 Massachusetts Medical Society. 


\section{University Library}

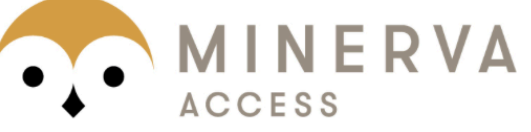

A gateway to Melbourne's research publications

Minerva Access is the Institutional Repository of The University of Melbourne

Author/s:

Bieber, T;Simpson, EL;Silverberg, JlThaci, D;Paul, C;Pink, AE;Kataoka, Y;Chu, C-

Y;DiBonaventura, M;Rojo, R;Antinew, J;Ionita, I;Sinclair, R;Forman, S;Zdybski, J;Biswas,

P;Malhotra, B;Zhang, F;Valdez, H

Title:

Abrocitinib versus Placebo or Dupilumab for Atopic Dermatitis

Date:

2021-03-25

Citation:

Bieber, T., Simpson, E. L., Silverberg, J. I., Thaci, D., Paul, C., Pink, A. E., Kataoka, Y., Chu, C. -Y., DiBonaventura, M., Rojo, R., Antinew, J., Ionita, I., Sinclair, R., Forman, S., Zdybski, J., Biswas, P., Malhotra, B., Zhang, F. \& Valdez, H. (2021). Abrocitinib versus Placebo or Dupilumab for Atopic Dermatitis. NEW ENGLAND JOURNAL OF MEDICINE, 384 (12), pp.1101-1112. https://doi.org/10.1056/NEJMoa2019380.

Persistent Link:

http://hdl.handle.net/11343/278531 